\title{
Potential Conflict of Interest Disclosures
}

It is the policy of the European Congress of Radiology to ensure balance, independence, objectivity, and scientific rigour in the congress programme. Knowledge of possible relationships with sponsors of any kind is mandatory in order to reinforce the educational and scientific message and to relieve any suspicion of bias.

Any potential conflict of interest involving ECR speakers should be made known so that the audience may form their own judgements about the presentation with a full disclosure of the facts. It is for the audience to determine whether the presenter's external interest may reflect a possible bias in either the work carried out or the conclusions presented.

In an optional survey of ECR 2012 Postgraduate Educational Programme and Scientific Session speakers, the individuals listed below disclosed the following relationships:

Abdullah B.J.J.: Research/Grant Support; Stempeutics

Antoch G.: Speaker; Siemens Healthcare, Bayer Healthcare, Novartis Oncology, Nordion.

Apfaltrer P.: Consultant; Dr. Schoepf receives research support from and is a consultant for Bayer, Bracco, General Electric, Medrad, and Siemens. Grant Recipient; Dr. Schoepf receives research support from and is a consultant for Bayer, Bracco, General Electric, Medrad, and Siemens.

Attenberger U.I.: Consultant; Bayer Healthcare.

Bamberg F.: Research/Grant Support; Bayer Healthcare (Germany). Speaker; Siemens Healthcare (Forchheim, Germany).

Bankier A.A.: Consultant; Spiration, Olympus Medical.

Barkhof F.: Advisory Board; Roche, Janssen Alzheimer Immunotherapy.

Bartling S.: Research/Grant Support; Support by Siemens Healthcare.

Baumhauer M.: CEO; Mint Medical GmbH.

Beyer T.: Founder; cmi-experts $\mathrm{GmbH}$.

Bock M.: Advisory Board; Siemens Clinical $7 \mathrm{~T}$ advisory board. Research/Grant Support; Research cooperation with Siemens.

Boellaard T.N.: Shareholder; Nuts Ohra Reasearch Fund.

Bongartz G.M.: Research/Grant Support; Bayer Healthcare (Switzerland).

Brancatelli G.: Speaker; Bayer Healthcare.

Cabrera T.: Employee; Florent Chandelier (co-author) is an employee of CADENS Imaging.

Cademartiri F.: Consultant; Bracco Imaging. Research/Grant Support; GE Healthcare.

Caramella D.: Equipment Support Recipient; Esaote.

Casselman J.: Consultant; Philips Healthcare, NewTom. Equipment Support Recipient; Philips Healthcare, NewTom. Speaker; Philips Healthcare.

Christe A.: Consultant; Guerbet. Research/Grant Support; Swiss Life.

Clevert D.A.: Speaker; Siemens, GE, Philips, Bracco.

Colosimo C.: Consultant; Bracco Diagnostics, Bayer Pharma. Speaker; Bracco Diagnostics, Bayer Pharma.

Desponds L.: Employee; Lionel Desponds: GE Healthcare; Bart Hoornaert: Philips Healthcare; Markus Lendl: Siemens Healthcare Sector.

Elliott S.T.: Advisory Board; Philips Healthcare. Speaker; Philips HealthCare, Hitachi Medical Systems, Bracco.
Fallenberg E.M.: Advisory Board; Bayer. Research/Grant Support; GE Healthcare, Siemens Healthcare, Bayer. Speaker; GE Healthcare, Siemens Healthcare, Bayer.

Froehlich J.M.: Consultant; Guerbet.

Frydrychowicz A.: Research/Grant Support; Scientific stipend Bracco Diagnostics. Other; The University of Wisconsin - Madison receives research support from GE Healthcare.

Fürst S.: Employee; B.W. Jakoby and R. Ladebeck (coauthors) are employees of Siemens.

Fütterer J.J.: Consultant; Galil Medical, Invivo.

Gallagher F.A.: Research/Grant Support; GE Healthcare.

Ganeshan B.: Shareholder; Balaji Ganeshan is a director and shareholder of TexRAD Ltd, an imaging software company that is developing a commercial version of the texture analysis considered in this study.

Geenen R.W.F.: Speaker; Symposium GE Healthcare, Symposium Bayer Healthcare.

Gilbert F.J.: Speaker; I have been paid travel expenses and speaker honorarium from Bracco.

Girard F.: Founder; Opteamage.

Gnant M.: Consultant; Merrion, Novartis. Research/ Grant Support; GSK, sanofi-aventis, Novartis, Roche. Speaker; Amgen, Pfizer, Novartis, GSK, Bayer, Sandoz, AstraZeneca, Genomic Health.

Goo J.: Consultant; Infinitt Healthcare Co.

Greenbaum N.: Research/Grant Support; the device manufacturer company named Activiews sponsored the study.

Halperin J.: Research/Grant Support; Toshiba Medical Systems.

Hansmann J.: Grant Recipient; Bayer Healthcare (Germany).

Harms S.E.: Consultant; Aurora Imaging Technology.

Helbich T.H.: Advisory Board; Philips, Siemens. Grant Recipient; Siemens, Founds Austrian National Bank.

Heussel C.: Board Member; Astellas, Gilead, MSD. Consultant; Schering-Plough, Pfizer, Basilea, Boehringer Ingelheim, Novartis, Roche, Astellas, Gilead, MSD. Equipment Support Recipient; ab medica / Actiview. Research/Grant Support; Novartis. Shareholder; Stada, GSK. Speaker; MEDA Pharma, Lilly, Bracco.

Hiwatashi A.: Employee; Makoto Obara.

Hohl C.: Consultant; WL Gore.

Hueper K.: Research/Grant Support; Abbott GmbH.

Hugg J.W.: Employee; СTO. 
Hunink M.G.M.: Author; Book: Decision Making in Health and Medicine. CUP 2001. Research/Grant Support; ZonMW, STW, NIH.

Hunold P.: Speaker; Bayer.

Idee J.: Employee; Guerbet.

Israel O.: Advisory Board; General Electric Healthcare. Investigator; UltraSPECT.

Jacobs C.: Research/Grant Support; MeVis Medical Solutions AG.

Johnson T.R.C.: Employee; U. Schuschnig (co-author) is an employee of Pari Pharma.

Kahn C.E.: CEO; Hotlight Inc.. Owner; Hotlight Inc..

Keller J.: Research/Grant Support; Czech government grants IGA-NT11328-4/2010 and MSM0021620816.

Kerl J.M.: Consultant; Siemens Healthcare AG. Speaker; Siemens Healthcare AG.

Kinkel K.: Investigator; study financed by Bayer Schering (Berlin, Germany).

Klotz E.: Employee; Siemens Healthcare.

Kolokythas O.: Advisory Board; Philips Healthcare. Equipment Support Recipient; Philips Healthcare. Grant Recipient; Philips Healthcare.

Kress B.: Research/Grant Support; Guerbet.

Krestin G.P.: Consultant; GEHC Europe. Grant Recipient; Bayer Healthcare, GEHC, Siemens. Research/Grant Support; Multiple.

Ladd M.E.: Research/Grant Support; Siemens Healthcare.

Laghi A.: Equipment Support Recipient; General Electric. Investigator; Guerbet, Promefarm. Speaker; Bayer, General Electric.

Lammer J.: Advisory Board; WL Gore, Abbott Vascular, Bosten Scientific International.

Lelieveldt B.: Research/Grant Support; FP7 ENCITE project.

Lin Z.: Employee; GE Healthcare.

Loewe C.: Board Member; member of the steering committee of the Apixaban studies. Investigator; principial investigator for studies sponsored by Guerbet and Bayer. Speaker; receiving speaker fees from Siemens, Bracco, GE Healthcare, Bayer, Guerbet, Cordis, Covidien.

Lonn L.: Board Member; www.mentice.com, www.orzone. com. Consultant; Cordis. Grant Recipient; COOK. Shareholder; www.orzone.com.

Mackay S.J.: Grant Recipient; College Of Radiographers Industry Partnership Scheme.

Mader C.: Research/Grant Support; GE Healthcare.

Mahnken A.H.: Consultant; Bayer, Siemens.

Malamateniou C.: Consultant; Philips Medical Hellas. Grant Recipient; BRC AHSC UK. Patent Holder; T1-weighted fetal brain MRI, 0913421.4, july 2009.

Mann R.M.: Speaker; Siemens Healthcare.

Marmarelis V.: Author; V. Marmarelis, M. Orme, M. Sofras, S. Hadjiagapis. Board Member; M. Orme, S. Hadjiagapis. CEO; S. Hadjiagapis. Consultant; G. Zografos. Employee; M. Sofras. Founder; V. Marmarelis, S. Hadjiagapis. Investigator; V. Marmarelis, M. Orme, M. Sofras, G. Zografos, D. Koulocheri. Owner; V. Marmarelis, S.
Hadjiagapis. Patent Holder; V. Marmarelis. Shareholder; V. Marmarelis, S. Hadjiagapis. Speaker; V. Marmarelis.

Martincich L.: Advisory Board; Breast MR GE 2010. Speaker; Bracco Imaging. Other; Blinded reader for Bracco Imaging and Bayer-Schering.

Mateka I.: Consultant; I will not have any benefit for presenting the results of the research programme carried out with General Electric (GE). However I was contracted and received money as consultant during this research.

Maurer M.H.: Consultant; Guerbet $\mathrm{GmbH}$.

Mc Entee M.F.: Advisory Board; Ziltron Ltd. Consultant; Ziltron Ltd.

Michaely H.J.: Advisory Board; Siemens Body MR. Consultant; Bayer. Speaker; Bayer, Siemens.

Moonen C.: Equipment Support Recipient; Philips Healthcare.

Morcos S.K.: Research/Grant Support; Guerbet. Speaker; Guerbet, Bayer, Bracco.

Najmi N.: Author; Houman Alizadeh, Neda Najmi, Mehrzad Mehdizadeh, Nooshin Najmi, Elham Taheri, Habib Mazaher.

Nappi J.J.: Other; Royalties, Hologic Inc.; Royalties, MEDIAN Technologies.

Nielsen M.: CEO; Biomediq. Founder; Biomediq. Grant Recipient; Synarc, Nordic Bioscience, Astra Zeneca. Shareholder; Biomediq.

Ntziachristos V.: Author; iThera Medical. Board Member; iThera Medical. Founder; iThera Medical. Patent Holder; iThera Medical. Shareholder; iThera Medical. Other; iThera Medical.

Nuyts J.: Patent Holder; Co-inventor on Siemens patent about PET/MRI attenuation correction. Research/Grant Support; Cooperation with Siemens on TOF-PET and breast tomosynthesis.

Ohno Y.: Research/Grant Support; Toshiba Medical Systems, Philips Healthcare, Bayer Pharma, Eizai Co. Ltd., DaiishiSankyo Co. Ltd.

O‘Leary D.: Employee; Lecturer University College Dublin, Ireland. Grant Recipient; Health Research Board of Ireland, Health Services Research grant 2006-2009.

Osborn A.G.: Author; Amirsys Publishing, Inc.. Board Member; Amirsys, Inc.. CEO; Amirsys Publishing, Inc.. Shareholder; Amirsys, Inc; Amirsys Publishing, Inc.. Speaker; Covidien, Inc..

Palkó A.: Advisory Board; Euromedic International Medical Advisory Board (chairman). Consultant; Covidien (educational consultant).

Panicek D.M.: Author; co-editor of handbook on Oncologic Imaging and Reporting; royalties from Elsevier.

Papanikolaou N.: CEO; N. Papanikolaou \& Associates.

Paul N.S.: Research/Grant Support; Toshiba Medical Systems.

Pedraza S.: Advisory Board; Member ECR committee about Undergraduate Radiology Education. Author; White paper of ECR about Radiology Education. Board Member; Editorial Board Stroke Journal. Consultant; Member Imaging Commitee DIAS trial. Investigator; Investigator Problem Based Learning on Radiology.

Pescatore J.: Employee; THALES company. 
Pirovano G.: Employee; Bracco Diagnostics, Inc.

Reinartz S.D.: Employee; T. Allmendinger (co-author) is employee of Siemens Healthcare (Forchheim, Germany).

Riccabona M.: Board Member; European Board for 3DUS / Kretz -GE. Research/Grant Support; Toshiba.

Robba T.: Employee; Valeria Clementi (co-author) is an employee of GE Healthcare Medical System.

Rovira-Cañellas A.: Advisory Board; NeuroTEC, BayerSchering Pharma, BTG International Ltd. Board Member; Am J Neuroradiol, Neuroradiology, European Society of Neuroradiology. Research/Grant Support; Bayer-Schering Pharma. Speaker; Bayer-Schering Pharma, Biogen Idec, Sanofi-Aventis, Merck-Serono.

Saam T.: Research/Grant Support; DFG, Diamed Medizintechnik, Bayer Schering AG.

Saxon S.R.: Research/Grant Support; Siemens.

Schaefer F.K.W.: Equipment Support Recipient; Supersonic Imagine (Aix en Provence, France).

Schäfers M.: Research/Grant Support; Siemens Medical.

Schindler A.: Consultant; U. Schoepf is a consultant for Bayer, Bracco, GE, Medrad, and Siemens. Employee; T. Allmendinger, S. Vogt and R. Raupach are employees of Siemens. Grant Recipient; U. Schoepf receives research support from Bayer, Bracco, GE, Medrad, and Siemens. Speaker; F. Bamberg receives speakers fee from Siemens.

Schmitt B.: Research/Grant Support; Siemens Healthcare.

Schneider G.K.: Investigator; BRACCO, Guerbet. Research/ Grant Support; Siemens. Speaker; BRACCO.

Schönberg S.O.: Other; The Institute of Clinical Radiology and Nuclear Medicine has research agreements with Siemens Healthcare Sector.

Schramm P.: Speaker; received speaker's honoraria from Siemens AG (Germany) and Bracco (Italy).

Schwenzer N.F.: Other; Technical Cooperation Contract Siemens.

Serafin Z.: Other; support from Bracco Imaging for ECR 2012 (travel, accommodation, registration fee).

Shaham D.: Consultant; Siemens AG.

Söderman M.: Speaker; Consultant Mindframe Inc, Scientific collaborations Philips Medicla Systems.

Sommer C.M.: Other; CeloNova Biosciences, San Antonio, USA, supported technically and financially the study described herein.

Steiger P.: Consultant; Johannes Fröhlich is working as a consultant for Guerbet.

Sutter R.: Other; V. Jellus and M. Nittka are employed by Siemens - they had no control of inclusion of any data and information that might present a conflict of interest.

Szikora I.: Consultant; Stryker Neurovascular, Covidien ev3. Equipment Support Recipient; Covidien ev3, Stryker Neurovascular. Grant Recipient; Hungarian State Research and Science Fund (OTKA).

Takx R.A.P.: Author; U.J. Schoepf. Consultant; BayerSchering, Bracco, General Electric, Medrad, Siemens, TeraRecon. Research/Grant Support; Bayer-Schering, Bracco, General Electric, Medrad, Siemens.
Taupitz M.: Research/Grant Support; Federal Ministry for Education and Research (BMBF, Germany): MAPIT I (support code 13N11091), German Research Foundation (DFG) TA 166/3-1, TA 166/3-2.

Thoeny H.C.: Grant Recipient; Swiss National Foundation for Research Grant No 320000-113512/1.

Tolan D.J.M.: Grant Recipient; Bracco. Speaker; Norgine.

Umutlu L.: Consultant; Bayer Healthcare.

Ursani A.: Research/Grant Support; Toshiba Medical Systems Canada.

Valvassori L.: Consultant; EV3, Balt, Concentric Medical, Codman Neurovascular, Terumo.

van Beek E.J.R.: Advisory Board; Siemens Medical Systems. CEO; Quantitative Clinical Trials Imaging Services. Owner; Quantitative Clinical Trials Imaging Services. Research/ Grant Support; Siemens Medical Systems, Toshiba Medical Systems. Speaker; Vital Images, Toshiba Medical Systems.

van den Boom R.: Research/Grant Support; Toshiba Medical Systems Corporation.

Vymazal J.: Investigator; Clinical Trial.

Wang C.: Patent Holder; Fast level set segmentation patent pending.

Weber M.: Research/Grant Support; This study was supported by a research grant from the "Deutsche Gesellschaft für Muskelkranke e.V"..

Werncke T.: Research/Grant Support; Federal ministry of education and research, Grant: 02NUK008D.

Wildberger J.E.: Research/Grant Support; Departmental: Bracco, Medrad, Philips Medical, GE, Siemens Medical Solutions. Speaker; Speaker's bureau: Bayer, Boston Scientific, GE, Siemens Medical Solutions.

Williams M.C.: Speaker; Lectured at courses sponsored by Toshiba.

Wintersperger B.J.: Research/Grant Support; Siemens Healthcare. Speaker; Siemens Healthcare, Bayer Healthcare.

Wirth S.: Research/Grant Support; General GE research grant without affecting the study in any way.

Zech C.J.: Advisory Board; Advisory Board Liver Imaging Bayer Healthcare. Research/Grant Support; Bayer Healthcare, Bracco Imaging, Siemens Medical Solutions. Speaker; Bayer Healthcare, Bracco Imaging.

Zhang D.: Employee; Toshiba America Medical Systems. 